\title{
Four new binary minor planets: (854) Frostia, (1089) Tama, (1313) Berna, (4492) Debussy ^
}

\author{
R. Behrend ${ }^{1}$, L. Bernasconi ${ }^{2,3}$, R. Roy ${ }^{2,4}$, A. Klotz ${ }^{5,6}$, F. Colas ${ }^{7,2}$, P. Antonini ${ }^{2,8}$, R. Aoun ${ }^{9}$, K. Augustesen ${ }^{10}$,
} E. Barbotin ${ }^{9}$, N. Berger ${ }^{11}$, H. Berrouachdi ${ }^{12}$, E. Brochard ${ }^{2}$, A. Cazenave ${ }^{9}$, C. Cavadore ${ }^{2}$, J. Coloma ${ }^{13}$, V. Cotrez ${ }^{9}$, S. Deconihout ${ }^{2}$, C. Demeautis ${ }^{2}$, J. Dorseuil ${ }^{12}$, G. Dubos ${ }^{9}$, R. Durkee ${ }^{14}$, E. Frappa ${ }^{15}$, F. Hormuth ${ }^{19}$, T. Itkonen ${ }^{17}$, C. Jacques ${ }^{31}$, L. Kurtze ${ }^{26}$, A. Laffont ${ }^{9}$, M. Lavayssière ${ }^{2}$, J. Lecacheux ${ }^{18}$, A. Leroy ${ }^{9}$, F. Manzinii ${ }^{20}$, G. Masi ${ }^{21,22}$, D. Matter ${ }^{2}$, R. Michelsen ${ }^{23}$, J. Nomen ${ }^{30}$, A. Oksanen ${ }^{24}$, P. Pääkkönen ${ }^{17}$, A. Peyrot ${ }^{12}$, E. Pimentel ${ }^{31}$, D. Pray ${ }^{25}$, C. Rinner ${ }^{2}$, S. Sanchez ${ }^{30}$, K. Sonnenberg ${ }^{16}$, S. Sposetti ${ }^{27}$, D. Starkey ${ }^{28}$, R. Stoss ${ }^{30}$, J.-P. Teng ${ }^{12}$, M. Vignand ${ }^{12}$, and N. Waelchli29

(Affiliations can be found after the references)

Received 28 June 2005 / Accepted 23 September 2005

\section{ABSTRACT}

Aims. We present evidence that four minor planets of the main belt are binary systems.

Methods. These discoveries are based on CCD photometric measurements made by many observers coordinated in a network of observatories. Results. Orbital and physical properties are derived from a total of 134 partial light curves involving 26 stations. (854) Frostia, (1089) Tama, (1313) Berna, and (4492) Debussy show mutual eclipses features on their light curves. In all cases, rotation and revolution are synchronous. Synodic periods are $37.728,16.444,25.464$ and $26.606 \mathrm{~h}$ respectively. From a simple model, we have derived their bulk densities as follows: $0.89 \pm 0.14,2.52 \pm 0.30,1.22 \pm 0.15$ and $0.91 \pm 0.10 \mathrm{~g} \mathrm{~cm}^{-3}$ respectively. Uncertainties in the bulk densities, arising from scattering and shadow effects are not taken into account. These could increase the density estimates by a factor up to 1.6. Our method of determining bulk density is completely independent of their mass and their diameter estimates. The low rotational periods and the low bulk densities clearly imply a collisional process to explain this kind of binary asteroid. Based on our database of a few thousand light curves of minor planets, the population of similar-sized objects in the main belt is estimated to $6 \pm 3$ percent in the $10-50 \mathrm{~km}$ diameter class.

Key words. planets and satellites: formation - minor planets, asteroids - techniques: photometric

\section{Introduction}

The first confirmed satellite of a minor planet was Dactyl around (243) Ida, discovered in 1993 on Galileo spacecraft images (Belton \& Carlson 1993). Ten years later, about fifty asteroids are suspected or confirmed to be multiple bodies. These asteroids are very useful in determining the physical and dynamical properties of small bodies, in particular the bulk density, which is important in understanding the collisional evolution of this kind of object.

Various techniques are used to detect satellites for minor planets: occultation of stars, light curves, direct imaging by spacecraft, radars or adaptive optic (see review by Merline et al. 2002). Binary studies allows the derivation of dimensions, shape, bulk densities, rotation and orbital properties of the bodies. Asteroid densities are known for only a few of them

^ Based on observations performed with the T1 $\mathrm{M}$ and $\mathrm{T} 60 \mathrm{~cm}$ at the Pic du Midi, TAROT at the Calern observatory, Faulkes-North at Hawaii, T50 $\mathrm{cm}$ at Brorfelde, and by many amateur observatories coordinated by Geneva Observatory. (see the review by Britt et al. 2002). We distinguish two types of multiple systems. Those consisting of comparable size bodies are designated as binary. The others are designated as primaries with satellites. When the orbital plane of a binary system is seen edge-on, mutual eclipses can be detected from light curves. That allows to discover binary systems that cannot be found by other methods. Based on binary system light curves anlysis, we present a method to derive the bulk density independently of size and mass determinations. From single opposition measurements the accuracy of the bulk density determination is about 30 percent, but this can be easily improved as in the case of (90) Antiope (Michalowski et al. 2004) by additional data from several oppositions.

This paper is devoted to the discovery of four binary systems from their light curves. Section 2 explains the observational method and the data reduction. In the Sect. 3.1 we determined the dimensions and the bulk densities. Finally, in Sect. 3.2, we estimate the proportion of main belt minor planets that are expected to be binary systems. 


\section{Observations and results}

\subsection{Observation strategy}

The less expensive, but time consuming method for discovering new binary systems of minor planets is light curve analysis. Nevertheless, the problem of observing time can be solved if numerous people at various places share their results. The main problem is the need for rapid analysis of data taken with heterogeneous instruments, because it is important to react rapidly when a light curve seems to be abnormal. All data from various observatories were collected by Behrend at Geneva Observatory. As soon as a new set of data is received, the corresponding light curve is updated. As the observers have neither the same telescopes, nor the same CCD cameras, the main problem is to merge data from different apparatus. This will be discussed in Sect. 2.4.

Mutual eclipse method is well adapted to detect binary systems, but for a photometry accuracy of $0.05 \mathrm{mag}$, the limit of the detectable radius ratio of the two components is about $2.5(3 \sigma)$. In this paper, we designate as binaries, systems where the ratio of the body sizes is within 2.5 .

\subsection{Observations}

The observations are based on series of CCD frames, taken during several nights. Each series is centred on the same field in order to derive differential magnitudes with the best possible accuracy. As no absolute magnitude calibration is needed by this method, images are usually not taken through filters. This allows the use of relatively small diameter telescopes.

We measured a typical magnitude dispersion less than 0.07 mag for stars brighter than $R=16$ th magnitude obtained with a $D=21.2 \mathrm{~cm}$ telescope. We consider that the small aperture telescopes $(D \sim 20 \mathrm{~cm})$ are well adapted to obtain 0.05 mag dispersion for minor planets brightest than $R=14$.

Most of the telescope diameters used by the observers, for the asteroids involved in this paper, lie between $21 \mathrm{~cm}$ and $60 \mathrm{~cm}$. We must emphasise that the discoveries were made with these small telescopes. We added some data from $1 \mathrm{~m}$ to $2 \mathrm{~m}$ telescopes. The time samplings are typically of the order of one minute.

\subsection{Images processing}

Usually, each observer processes raw images using bias, dark and flat frames. Then an algorithm of flux measurement is used to extract the flux of the asteroid and some comparison stars in the field. Fluxes are measured for each image. Uncertainty is evaluated with the help of a test star, or computed during measurement. Various photometric softwares were used (Prism, AudeLA, Photo, etc.) and algorithms of flux extraction are mostly based on profile fitting. At the end of the processes, the data is written in a formatted text file and sent to the Geneva Observatory.

\subsection{Merging light curves}

The method of merging data to a single light curve is described in Behrend (2001). The goal is to fit data with the harmonic Eq. (1) below:

$m(t)=c_{1}+\sum_{j=1}^{J}\left(a_{j} \cos \left(2 \pi j \frac{t-E}{T}\right)+b_{j} \sin \left(2 \pi j \frac{t-E}{T}\right)\right)$.

Here $J$ is the order of the highest harmonic. $E$ is the 0 -phase epoch, $t$ is the date - both corrected for asteroid to observer light travel time - and $T$ the synodic period. $T$ is found by scanning trial values and minimizing the quadratic residue. The $J$ order is chosen so as not to introduce artefacts. Before the Fourier analysis is done, distances and phase effects are removed by subtracting from each observation the $V$-magnitude given by the ephemeris. Colour indexes are not taken into account at this stage.

The main problem to merge data is that the constant $c_{1}$ is not necessarily the same from a set to another one. This is due to the various cameras, telescopes and reference stars used. This problem is solved by the determination of $c_{\mathrm{k}}$ for each data set to be merged. This is equivalent to an offset of all magnitudes in each set to give the data the same mean value.

At the end of the process, data are plotted against orbital phase, as shown in Figs. 1 and 3.

\subsection{Results}

About 450 asteroids were measured since Jan. 2000 by observers collaborating with Geneva Observatory. Amongst them, from Dec. 2003 to Jul. 2004, four minor planets were found to exhibit eclipse features and were announced in IAU circulars: (1089) Tama (Behrend et al. 2004a), (1313) Berna (Behrend et al. 2004b), (4492) Debussy (Behrend et al. 2004c) and (954) Frostia (Behrend et al. 2004d). How we discovered the particular status of Tama and Berna is explained in Behrend et al. (2004e).

About ten other asteroids exhibit non-usual features in their light curve, but complementary observations are required to identify their binarity.

We would like to emphasize that a helpful method to identify binarity is to use the polar plot of the flux. In the traditional phase-magnitude diagram, a binary synchronised system is characterized by a relatively small bend. However, a plot of flux in polar coordinates, like Fig. 4, reveals two opposed $\mathrm{V}$-shapes which provides a strong signature for binarity.

\section{Analysis}

The four minor planets of the title of this paper show some common features in their light curves (Fig. 2): i) the rotational light curve has a continuous quasi sinusoidal variation due to the non spherical shape of the bodies; ii) the eclipsing light curve seen as a pronounced $\mathrm{V}$-shape feature due to mutual eclipses. These kinds of light curves are well known in the eclipsing variable stars (see Kopal 1990). 

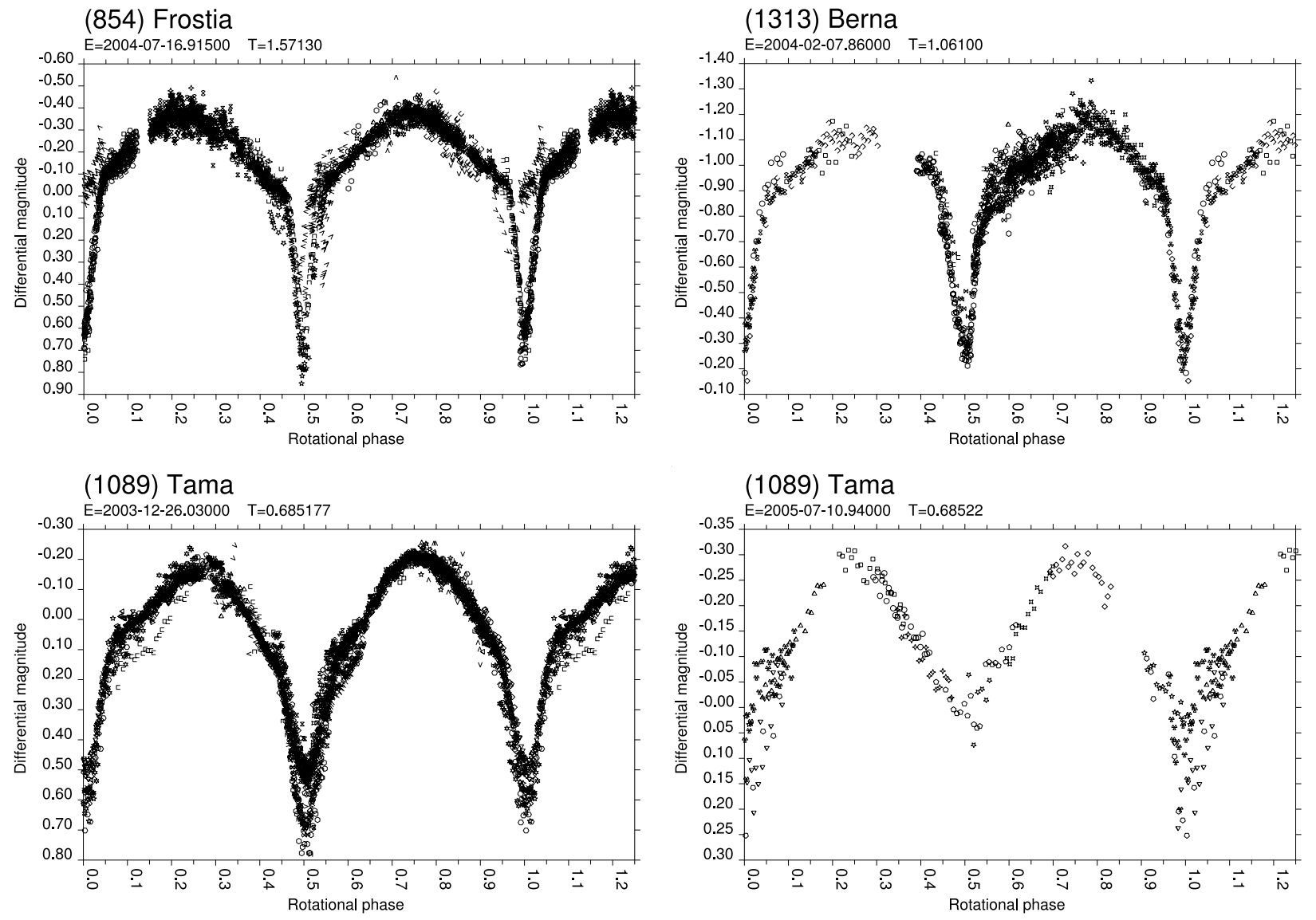

Fig. 1. Phased light curves for all observations for three of the four minor planets of this study - see Fig. 3 for the last one. $E$ is the epoch in UTC "onboard" the asteroid corresponding to the origin of the phase plot. $T$ is the adopted synodic period for folding the observations. The horizontal axis represents the time of the observations, corrected for light travel, since $E$, in units of $T$, modulo 1 ; the leftmost quarter is repeated at right. The vertical axis represents the magnitude, usually unfiltered, based on the $R$-band of the USNO-A2.0 star catalogue (Monet 1998), from which the $V$-magnitude from the ephemeris is subtracted - used photometric parameters are tabulated in Table 1. One can easily notice some changes in the profile of the mutual phenomenons during the months following the discoveries, due to the evolution of the Sun-Asteroid-Earth configuration.

\subsection{Size, orbit and densities}

For a first approach, as the amplitude of eclipses does not show any large parity changes nor any plateau at minimum, the two components are believed to be about the same size. An other index that supports to this conclusion is the depth value of the eclipses. A total eclipse, at phase angle 0 , of two bodies of same size and same albedo should give a depth $\Delta e=$ $2.5 \log _{10} 2=0.75 \mathrm{mag}$. The four asteroids exhibit eclipses higher than 0.5 mag which gives a ratio for equivalent radius 1.6 at the most.

We build a theoretical model to extract orbital and physical parameters for the bodies from photometric properties during eclipses. Hypothesises are: i) the two bodies are prolate ellipsoids of same size with greatest radius pointing toward each other $\left(r_{\mathrm{a}}>r_{\mathrm{b}}\right)$; ii) the orbit is circular and orbital period is locked to the rotational period; iii) the densities are the same for the two bodies. See Fig. 5 for a schematic description.

From $\Delta m$, we derived the $r_{\mathrm{b}} / r_{\mathrm{a}}$ axis ratio for prolate ellipsoids. We used the method described in Kaasalainen \& Torppa (2001) to compute the relation that links the $r_{\mathrm{b}} / r_{\mathrm{a}}$ ratio to $\Delta m$. In the case of the generally accepted Lommel-Seeliger
Table 1. Bibliographic parameters of the four asteroids of this study. MB means Main Belt. $H$ and $G$ magnitudes parameters are taken from the Minor Planet Center database, 2004. Group, family, albedo and taxonomic class are taken in Bendjoya et al. (2002) and references therein. Cells with n.d. means no data.

\begin{tabular}{ccccc}
\hline \hline & $(854)$ & $(1089)$ & $(1313)$ & $(4492)$ \\
& Frostia & Tama & Berna & Debussy \\
\hline$H$ & 12.1 & 11.6 & 11.8 & 12.9 \\
$G$ & 0.15 & 0.15 & 0.15 & 0.15 \\
Group & MB I & Flora & MB II & MB IIb \\
Kozai family & 9 & 1 (Flora) & 27 (Io) & n.d. \\
Taxonomic class & n.d. & S & n.d. & n.d. \\
Albedo & n.d. & 0.244 & n.d. & n.d. \\
& & \pm 0.026 & & \\
\hline
\end{tabular}

scattering law, it gives:

$r_{\mathrm{b}} / r_{\mathrm{a}}=10^{-0.4 \Delta m}$.

Harris \& Lagerros (2002) give the formula to convert the $H$ absolute magnitude to the diameter of a spherical body. We scaled their formula to give the equivalent radius $r_{\mathrm{eq}}$ for each 
Table 2. Measured parameters of the four asteroids of this study. $l$ and $b$ are respectively the ecliptic heliocentic longitude and latitude. $T$ represents the synodic period. $\Delta m$ denotes the rotational amplitude. The eclipsing amplitude and duration are respectively designed by $\Delta e$ and $\Delta \phi$.

\begin{tabular}{|c|c|c|c|c|c|c|c|}
\hline & $\begin{array}{c}(854) \\
\text { Frostia }\end{array}$ & \multicolumn{2}{|c|}{$\begin{array}{c}(1089) \\
\text { Tama }\end{array}$} & $\begin{array}{l}(1313) \\
\text { Berna }\end{array}$ & \multicolumn{3}{|c|}{$\begin{array}{c}(4492) \\
\text { Debussy }\end{array}$} \\
\hline First date & $2004-07-16$ & $2003-12-26$ & 2005-07-01 & 2004-02-06 & $2002-10-30$ & 2004-03-18 & $2005-04-30$ \\
\hline Last date & $2004-10-21$ & 2004-05-21 & 2005-08-11 & 2004-03-10 & $2002-12-04$ & 2004-05-18 & 2005-04-30 \\
\hline$l\left(^{\circ}\right)$ & $303-337$ & $100-151$ & $255-265$ & $117-125$ & $35-46$ & $164-175$ & 230 \\
\hline$b\left(^{\circ}\right)$ & $5.6-3.3$ & $1.8-3.7$ & $-0.2--0.9$ & $0.4--1.4$ & $5.7-6.7$ & $0.8--0.7$ & -6.9 \\
\hline$T(\mathrm{~h})$ & 37.728 & 16.444 & 16.445 & 25.464 & & 26.606 & \\
\hline & \pm 0.001 & \pm 0.001 & \pm 0.005 & \pm 0.001 & & \pm 0.001 & \\
\hline$\Delta m$ (mag) & $\begin{array}{c}0.37 \\
\pm 0.01\end{array}$ & $\begin{array}{c}0.41 \\
\pm 0.01\end{array}$ & $\begin{array}{l}0.28 \\
0.02\end{array}$ & $\begin{array}{c}0.28 \\
\pm 0.02\end{array}$ & $\begin{array}{c}0.39 \\
\pm 0.01\end{array}$ & $\begin{array}{c}0.48 \\
\pm 0.01\end{array}$ & $>0.3$ \\
\hline$\Delta e(\mathrm{mag})$ & $\begin{array}{c}0.80 \\
\pm 0.03\end{array}$ & $\begin{array}{c}0.55 \\
\pm 0.03\end{array}$ & $\begin{array}{c}0.15 \\
\pm 0.05\end{array}$ & $\begin{array}{c}0.75 \\
\pm 0.05\end{array}$ & $\begin{array}{c}0.65 \\
\pm 0.03\end{array}$ & $\begin{array}{c}0.65 \\
\pm 0.03\end{array}$ & $>0.5$ \\
\hline$\Delta \phi$ (phase) & $\begin{array}{c}0.095 \\
\pm 0.005\end{array}$ & $\begin{array}{c}0.115 \\
\pm 0.005\end{array}$ & & $\begin{array}{l}0.115 \\
\pm 0.01\end{array}$ & $\begin{array}{c}0.115 \\
\pm 0.005\end{array}$ & $\begin{array}{c}0.12 \\
\pm 0.01\end{array}$ & $\sim 0.12$ \\
\hline Total of series & 34 & 36 & 12 & 27 & 6 & 18 & 1 \\
\hline
\end{tabular}

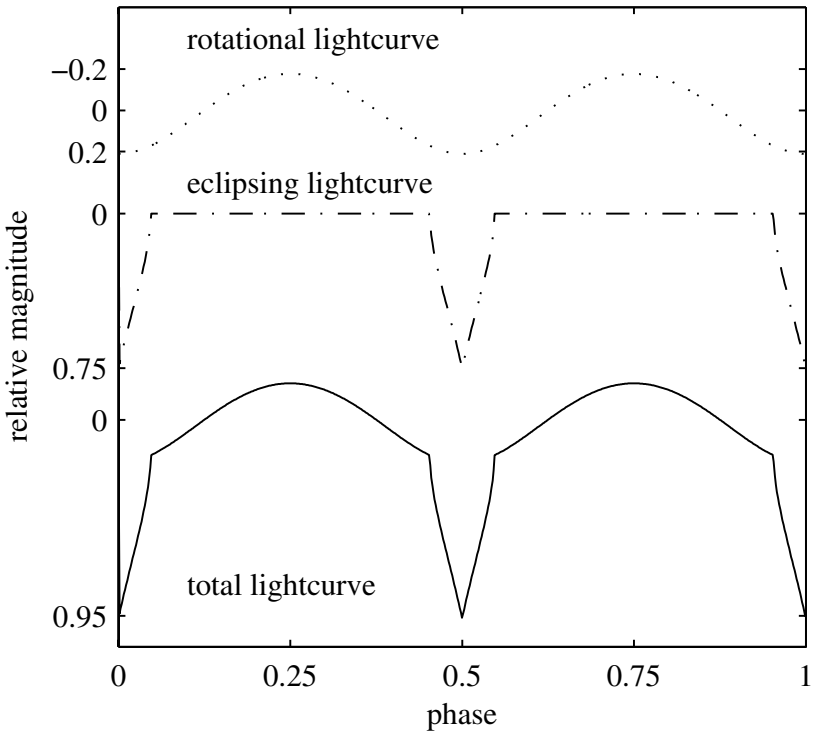

Fig. 2. This plots show the theoretical light curve of a binary system consisting of two equal sized prolate bodies $(10 \times 7 \times 7 \mathrm{~km})$ separated by $50 \mathrm{~km}$. The orbit is circular and seen edge-on. Top: the rotational light curve is the component due to the rotation of the prolate bodies, characterized by the amplitude $\Delta m$. Middle: the eclipsing light curve is flat outside eclipses and exhibits V-shape features with an amplitude of $\Delta e(0.75 \mathrm{mag}$ in case of total eclipses) and an eclipse duration $\Delta \phi$. Bottom: the total light curve is the combination of the rotational and eclipsing light curves.

body:

$r_{\mathrm{eq}}=\frac{H_{0} 10^{-0.2 H}}{\sqrt{8 p}}$

where $H_{0}=1329 \mathrm{~km}, p$ is the geometric albedo. Then, $r_{\mathrm{a}}$ and $r_{\mathrm{b}}$ can be computed:

$r_{\mathrm{a}}=r_{\mathrm{eq}} \sqrt{r_{\mathrm{a}} / r_{\mathrm{b}}}$
$r_{\mathrm{b}}=r_{\mathrm{eq}} \sqrt{r_{\mathrm{b}} / r_{\mathrm{a}}}$.
Geometrical considerations allow to link the semi-major axis $a$ to $\Delta \phi$, the relative duration of one eclipse expressed in angular form:

$a=\frac{r_{\mathrm{b}}}{\sin (\Delta \phi / 2)}$.

Then the mass $M$ of a body (considered as point-like, for simplicity) can be expressed via the third Kepler law:

$M=\frac{16 \pi^{2} a^{3}}{G T^{2}}$

where $G$ is the gravitation constant and $T$ the sideral revolution period. We took sideral periods equal to synodic ones because differences are not important for this study. The density $\rho$ is:

$\rho=\frac{M}{\frac{4}{3} \pi r_{\mathrm{a}} r_{\mathrm{b}}^{2}}$.

In the case of a Lommel-Seeliger scattering law, the density can be rewritten using only measured parameters:

$\rho=\frac{12 \pi}{\mathrm{G}} \frac{1}{T^{2}} \frac{10^{-0.4 \Delta m}}{\sin ^{3}(\Delta \phi / 2)}$

Uncertainties in $\rho$ come essentially from the $\sin ^{3}(\Delta \phi / 2)$ term. It is hard to identify breaks of eclipses in the light curves with a very good accuracy. Typically, an error of \pm 0.005 for $\Delta \phi \simeq 0.1$ leads to an uncertainty of about $\pm 30 \%$ on the density. Moreover, when eclipses are not total, $\Delta \phi$ is underestimated. Nevertheless, in the worst case (Tama, $\Delta e=0.55$ ) the $\rho$ value must be multiplied by factor 0.96 . In conclusion, the non-total eclipses have only weak influence on the density determination.

An extra uncertainty comes from the scattering law. We used the ideal Lommel-Seeliger law as discussed above. In the case of pure Lambertian scattering law, the $\rho$ values must be multiplied by factor $10^{0.15 \Delta m}$. As values of $\Delta m$ lie in the range $0.28-0.37$, it implies that $\rho$ must be multiplied by a factor from 1.10 to 1.18 to give upper limits on the density. It must be noticed that models generally fit observations by pure Lommel(4) Seeliger or a mix with Lambert but never a pure Lambert law. 

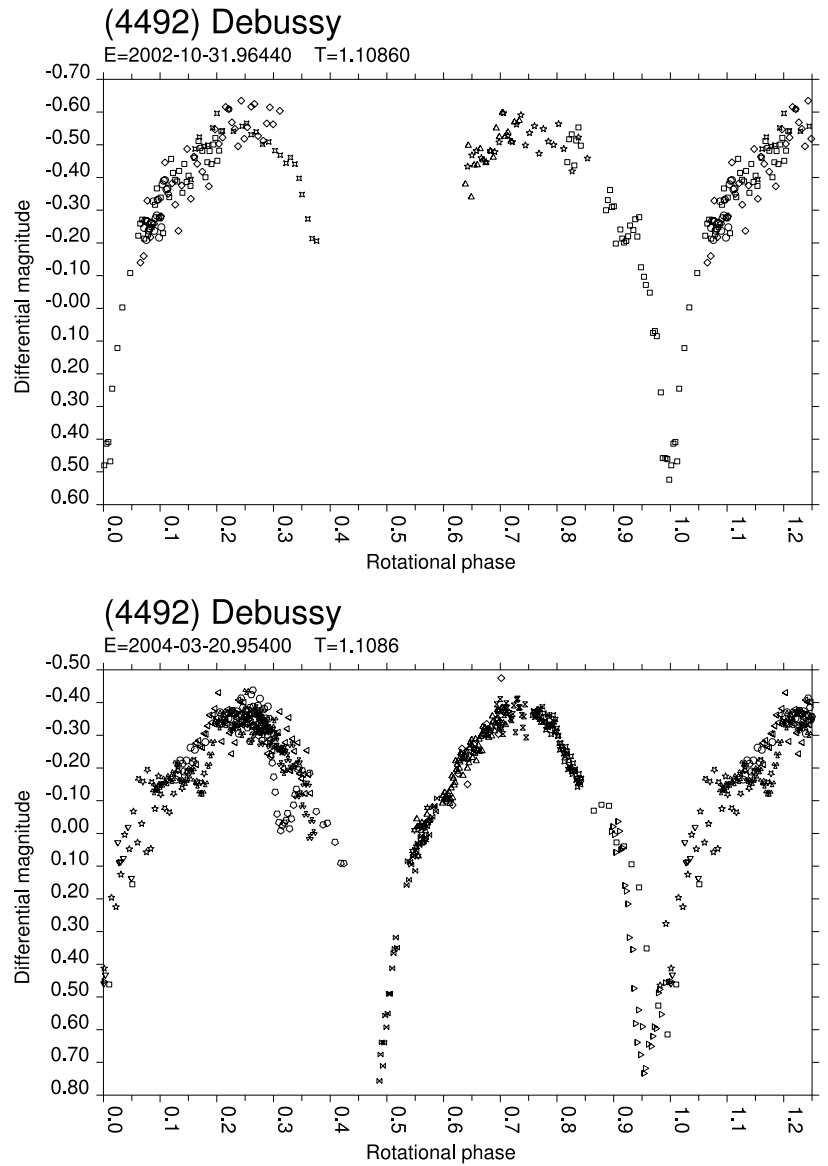

(4492) Debussy

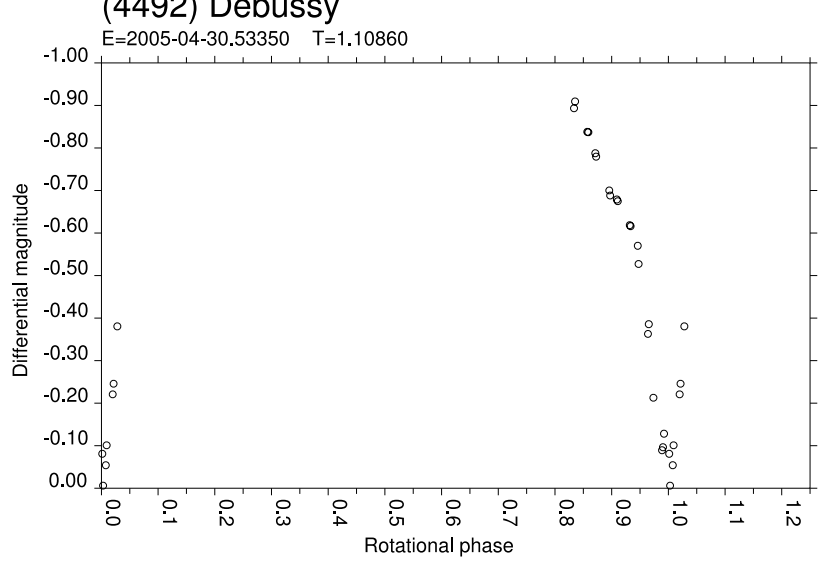

Fig. 3. Phased light curves for (4492) Debussy. See explanations in Fig. 1.

Another uncertainty comes from the shadow projected by one body onto of the other. Outside the opposition date, this effect can be important. Leading or trailing shadows lead to over-estimates of the $\Delta \phi$ value. An over-estimation of $\Delta \phi \simeq 0.1$ by $20 \%$ leads to an increase in $\rho$ by a factor of 1.6. The shadow effect can be included in models when the pole axis direction is known. This is not the case for the measurements presented in this paper.

We computed quantitatively the tidal effects on the prolateness of the bodies using the formula for tidal distortion

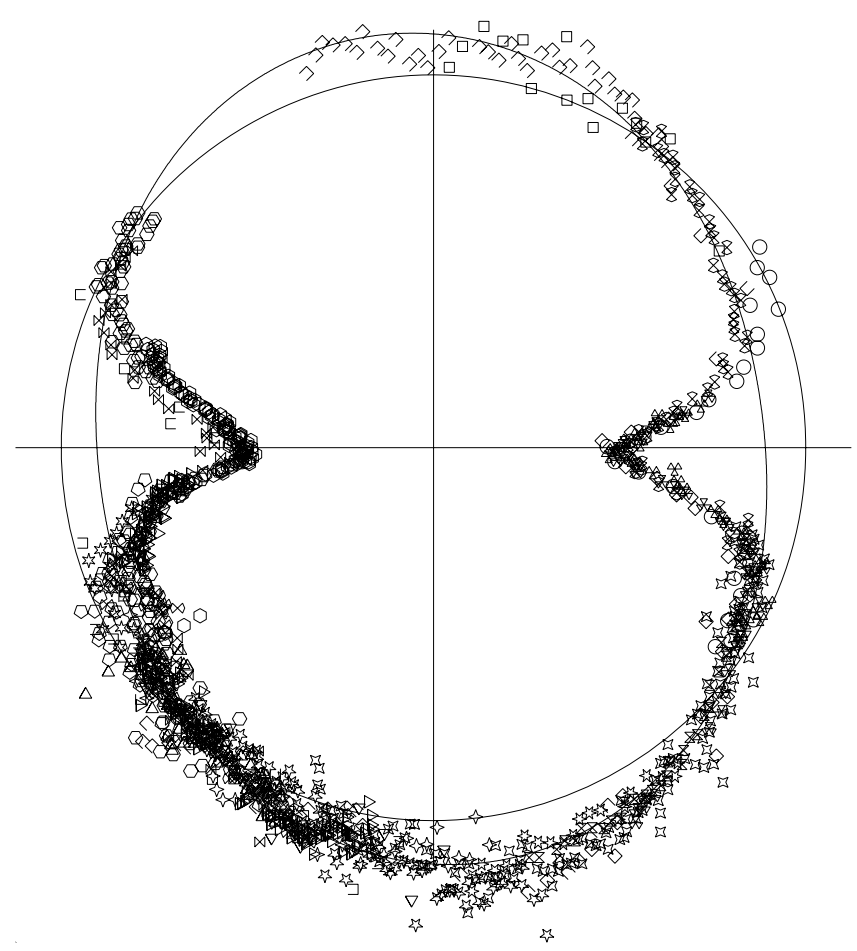

Fig. 4. Polar plot of the intrinsic variations of the flux (linear scale) of the asteroid Berna during one rotation of the system - radial coordinate. The angular coordinate is the phase $\frac{t-E}{T}$. The ellipsoid is the computed light curve from the observations out of the eclipses. The occultation feature shows strongly compared with a traditional light curve.

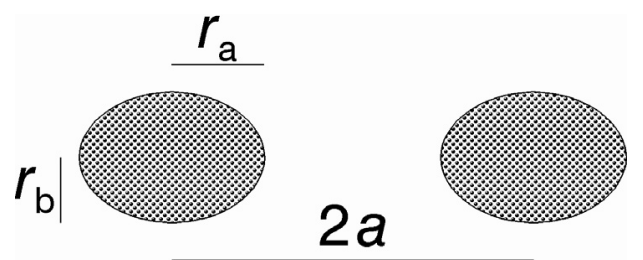

Fig. 5. Geometry and symbol definitions used in the model described in Sect. 3.1.

of a liquid sphere under the action of a distant point mass (Danby 1992):

$1-\frac{r_{\mathrm{b}}}{r_{\mathrm{a}}}=\epsilon=\frac{45}{16 \pi} \frac{M}{a^{3} \rho}$.

Table 3 summarizes the computed values taking account of the uncertainties except for those concerning the scattering law and shadow effects. The albedos were taken to be $p=0.15 \pm 0.10$ when they are not known and $H$ with an uncertainties of \pm 0.05 . Note that $\rho$ is independent of both the albedo and the $H$ values. On the contrary, the uncertainty on albedo is preponderant for the determination of the sizes and even more for the masses. Only (1089) Tama has a size derived from IRAS measurements, so a good estimation of its albedo, therefore of its mass and its size.

The $a / r_{\mathrm{a}}$ ratio is near the Roche limit. As a consequence of tidal effects, the ratio $r_{\mathrm{b}} / r_{\mathrm{a}}$ is correlated to $1-\epsilon$ values. 
Table 3. Computed parameters of the four asteroids. The two bodies are considered as prolate ellipsoids of same size with greatest radius pointing toward each other. Their orbits are assumed to be circular and orbital period is synchronized to the rotational period. Uncertainties does not include the shadow effects which can increase the semi-major axis, mass and density values.

\begin{tabular}{ccccc}
\hline \hline & $(854)$ & $(1089)$ & $(1313)$ & $(4492)$ \\
& Frostia & Tama & Berna & Debussy \\
\hline$r_{\mathrm{a}}(\mathrm{km})$ & $4.08-9.65$ & $5.08-5.99$ & $4.48-10.7$ & $2.99-7.09$ \\
$r_{\mathrm{b}}(\mathrm{km})$ & $2.95-6.99$ & $3.48-4.11$ & $3.54-8.39$ & $1.92-4.56$ \\
$a(\mathrm{~km})$ & $9.57-25.0$ & $9.47-12.2$ & $9.63-24.8$ & $5.23-13.5$ \\
$M\left(10^{13} \mathrm{~kg}\right)$ & $11-201$ & $57-121$ & $25-426$ & $3.7-63$ \\
$\rho\left(\mathrm{g} \mathrm{cm}^{-3}\right)$ & $0.75-1.02$ & $2.23-2.82$ & $1.07-1.36$ & $0.80-1.01$ \\
$r_{\mathrm{b}} / r_{\mathrm{a}}$ & 0.724 & 0.685 & 0.790 & 0.643 \\
$a / r_{\mathrm{a}}$ & $2.34-2.59$ & $1.86-2.03$ & $2.14-2.33$ & $1.74-1.90$ \\
$a / r_{\mathrm{b}}$ & $3.23-3.59$ & $2.71-2.95$ & $2.71-2.96$ & $2.71-2.96$ \\
$1-\epsilon$ & $0.84-0.89$ & $0.72-0.79$ & $0.76-0.82$ & $0.71-0.77$ \\
\hline
\end{tabular}

We conclude that tidal effects are the main processes that explain the "prolateness" of the bodies.

(854) Frostia and (4492) Debussy have the lightest densities in this study, possibly lower than that of the water ice. (1313) Berna is just denser than water ice. We consider the lightest one, (854) Frostia, with a mean bulk density of 0.9 . This is comparable with the C Type asteroid (90) Antiope with a bulk density of 1.3 (Michalowski et al. 2004). If it is a $C$ type asteroid, the bulk density of its matter could be 1.65 like the CI Orgueil meteorite. So a bulk density of 0.9 can be reached with a macroporosity of 45 percents. This is one of the highest value but still compatible with a model of rubble pile loosely consolidated (Britt et al. 2002). (1089) Tama is a S type asteroid (Bendjoya et al. 2002), its measured bulk density of 2.5 is comparable to those of (243) Ida with a macroporosity of 20 percents.

These considerations raise the question of the origin of the binaries. Is (1089) Tama the result of a capture mechanism, and are the three others the fruit of a collision mechanism? The energy in such a process has to be enough to totally disrupt the parent bodies, but not too high to prevent reformation of the bodies from the mixture of rubble. That could explain the similarities in the sizes, separations and orbital periods in (854) Frostia, (1313) Berna and (4492) Debussy. Spectroscopic observations of both bodies during total eclipses would allow the determination of the taxonomic class of each component; we believe that bodies of different classes are possible only in a capture process. Thus we predict that spectroscopy will confirm that each component of the binary has the same spectroscopic class.

We have not enough data to constrain a more complex model. Light curves obtained at the next oppositions should allow us to include different radius for each component and the inclination of orbital planes. An interesting effect can be seen on the first eclipse of the (1089) Tama light curve (Figs. 1 and 3). Measurements, obtained when phase angle came larger, exhibit some changes indicating a shadow effect of the forward component onto the backward one. Such phenomenon are expected to greatly constrain the orbital model. (1089) Tama was observed on 1998-04-04 by Sárneczky, Szabó \& Kiss (1999). Their light curve, covering about a quarter of the period, including the minimum of brightness, did not show any sign of eclipse in the 0.03 mag variation. The orbital plane of the components of (1089) Tama are therefore probably far from the ecliptic. The opposite situation is present with (4492) Debussy, for which three successive oppositions are marked by strong eclipses. Such phenomenon are probably present during the whole revolution around the Sun. For (1313) Berna and (854) Frostia, we are not aware of other light curves.

Follow-up of these binary asteroids during the next apparitions is necessary to determine a reliable model for each asteroid, including orbital and geometrical quantities, the density and some photometric parameters about the surfaces, by fitting such a model to the observations.

\subsection{About the binary population}

\subsubsection{Description of the sample}

Amongst the $4.5 \times 10^{2}$ observed asteroids in our database, about 10 of them were observed knowing that they were binary (for example (69230) Hermes, Margot et al. 2003; (90) Antiope, Michalowski et al. 2004), whilst others were observed without detecting that they are in fact a minor planet plus one satellite (for example (130) Elektra, (45) Eugenia, (22) Kalliope). About 40 other minor planets cannot be included in the binarity detection because they were not observed for long enough to cover at least $2 / 3$ of the phased light curve (at least, half the light curve is required to unambiguously detect an eclipse event in a synchronised system). This leaves about $4.0 \times 10^{2}$ asteroids to perform a statistical study. The selection criteria of these minor planets is only biased by the magnitude that can be reached using small telescopes. Figure 6 displays the histogram of the corresponding $H$ magnitudes. Our study concerns radius lying between 10 and $50 \mathrm{~km}$. It must be added that less than $5 \%$ of the sample was observed at two oppositions.

Only four asteroids of this study were recognized as binaries by their eclipse behaviours. Some others are suspected to be also binaries, but as they do not reach the detection criteria, we did not count them in the present statistic. We have not used data from other data bases because statistic interpretation is difficult due to different selection biases. 


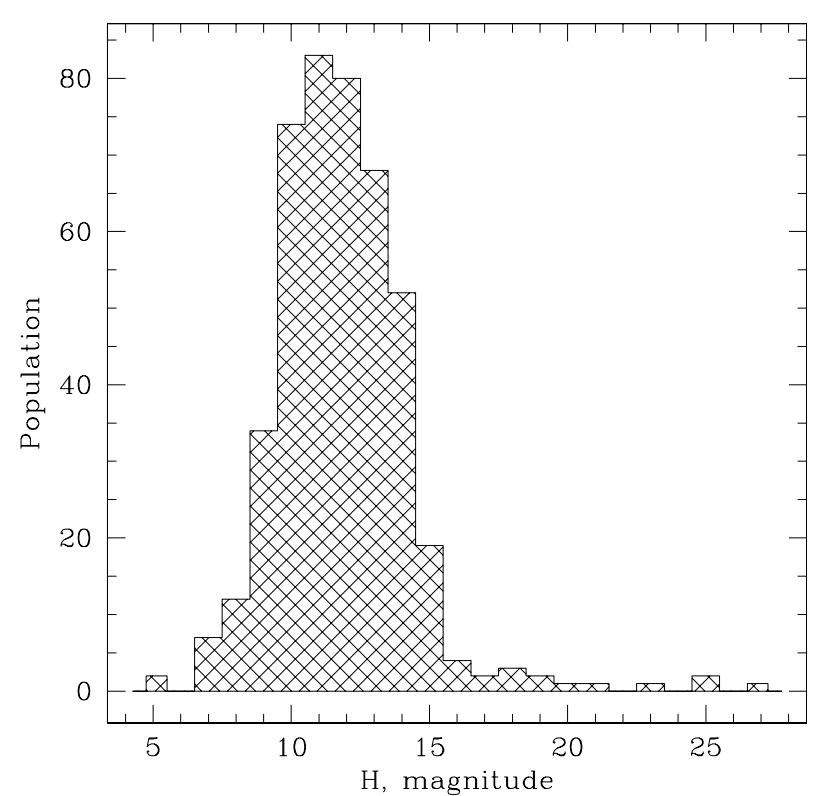

Fig. 6. The completed distribution of the $4.5 \times 10^{2}$ asteroids in our survey, as a function of their $H$ absolute magnitude parameter. For most objects of this survey, $9<H<15$ roughly corresponding to diameters from 10 to $50 \mathrm{~km}$. A few near Earth objects with $H>17$ and some big main belt asteroids with $H<7$ are also present.

\subsubsection{The model}

Knowing that only four targets of the $4.0 \times 10^{2}$ asteroid sample were detected as binaries, what is the proportion of these asteroids? As all the binaries of this study were found near opposition, one can derive some simple calculations to estimate the proportion of synchronized binary asteroids among the other ones.

An eclipse can be detected if the latitude of the Sun/Earth, in the sky of the asteroid, is in absolute value below a certain value, $\zeta$. This value depends on the observer's ability to distinguish a mutual event from features commonly found in light curves of small, non-spherical asteroids. We computed theoretical eclipse profiles for equal sized binaries, and estimated that a 0.3 mag depth is the practical limit of detection, not due to its depth, but due to its shape. This corresponds approximately to an eclipse in which the centre of one body grazes the other body.

For an inclination $i$ of the plane of rotation of a binary system, the relative part of a circular orbit around the Sun for which the eclipses are detectable, according to the previous criteria, at opposition, near phase angle 0 , is given by the following function:

$\tau(i, \zeta)=\frac{\varphi(i, \zeta)}{\pi / 2}$

$\varphi(i, \zeta)$ is the ecliptic longitude, from the line of nodes, of the end of the arc in which the eclipses are detectable. The corresponding formulation for this angle is:

$\varphi(i, \zeta)=\left\{\begin{array}{lc}\pi / 2 & \text { if } i \leq \zeta \\ \arcsin \frac{\sin \zeta}{\sin i} & \text { else. }\end{array}\right.$

Assuming that the spin axis of the binary systems are homogeneously distributed on the celestial sphere, the proportion of binary systems which can be detected among their reservoir, at a given opposition, is estimated by

$p(\zeta)=\frac{\int_{0}^{\pi / 2} \tau(i, \zeta) \sin i \mathrm{~d} i}{\int_{0}^{\pi / 2} \sin i \mathrm{~d} i}=\int_{0}^{\pi / 2} \tau(i, \zeta) \sin i \mathrm{~d} i$.

Equation (12) does not include any factors depending on the time interval between the first and last night of observation. This is motivated by the fact that during the 1-2 weeks interval typically used for main belt asteroids at opposition, the changes in the geometric configuration are small in comparison with $\zeta$. This is not the case for near Earth objects for which a more complete formula is required. If we admit the validity of the detection criteria given above, and a mean value $\frac{r_{\mathrm{b}}}{2 a} \sim \frac{1}{6}$, we can compute

$p\left(\zeta=\frac{1}{6}\right) \sim 0.17$.

This means that only $17 \%$ of the binary asteroids that have a ratio $r_{\mathrm{b}} /(2 a) \sim 1 / 6$ can be detected at a given opposition. As four asteroids were recognized as binary, about $4 / 0.17 \simeq 24$ of the total $\left(4.0 \times 10^{2}\right)$ should be of the same type. Taking account of the uncertainties, the proportion of binary systems in the main belt is thus probably around $6 \pm 3$ percents.

Now a question rises: why have we found so much binary asteroids in our survey, since thousands of light curves have been observed in the past? In the past, observers measured several objects in the night with a photometer, so the time sample was generally large and an eclipse due to an occultation could be missed or simply sampled by 1 or 2 points and then considered as bad data. For our survey, we used small size telescopes. To get good data we observed the same object all the night, so occultation features could not be missed or miss interpreted.

\section{Conclusion}

We have presented four new binary systems that are composed, as a first approximation, by twin prolate ellipsoidal components $\left(r_{\mathrm{b}} / r_{\mathrm{a}} \sim 0.7\right)$. Orbital motion is close to circular and the semi major axis is 1.7 to 2.6 times $r_{\mathrm{a}}$.

All of these systems have synchronized orbital and rotation periods, well larger than the mean rotational periods $(8 \mathrm{~h})$ of the main belt asteroids. This remark is an important criterion to find other binary systems from light curves. We show that the polar projection of the light curve allows easy identification of new binary systems with a good confidence level.

Only (1089) Tama of the Flora family has a density of stony bodies $\left(2.5 \mathrm{~g} \mathrm{~cm}^{-3}\right)$. The three other asteroids have densities lower than $1.4 \mathrm{~g} \mathrm{~cm}^{-3}$, probably even lower than $1 \mathrm{~g} \mathrm{~cm}^{-3}$ for (854) Frostia and (4492) Debussy. The two last asteroids are presently amongst the lowest bulk density bodies known in the main belt. This density can be interpreted by a material with an high microporosity (35\%) similar to CI Meteorites and a macroporosity of $45 \%$. This macroporosity is very high. It can only be interpreted by a rubble pile without cohesion forces other than gravity. But water ice in the interior of the asteroid is still possible. Moreover this ice can be a glue for the asteroid. 
From the large number of asteroids studied, more than $4 \times 10^{2}$, we used statistical criterion to estimate that twin asteroids could represent $6 \pm 3 \%$ of the total number of minor planets in the main belt with radius of about $10 \mathrm{~km}$. The probability for photometric discovery of such a binary asteroid is, at a given opposition, of the order of $17 \%$. This probability is for binary asteroids with components of about the same size. It is clear that the total probability of multiple asteroids is much greater. This problem could be investigated by numerical models of collisions.

Systematic spectroscopic observations of binary asteroids during total eclipses is certainly a good way for a better understanding of the origins of these asteroids. We predict that in case of collisional origin, the two components of each will be found to have the similar spectral class.

It will be now important to perform a follow-up of the four minor planets presented here at the next oppositions and also when phase angle is large in order to analyze the shadow effects on the light curves and to determine albedo independently. This last point should help to invert light curves more efficiently, bringing a significant gain in the determination of their bulk densities.

Acknowledgements. We thank the AUDE association support to allow motivate new members and to communicate quickly between observers.

We also thank A. Harris and P. Pravec for numerous discussions about binary asteroids. Thanks are also expressed to J.-L. Margot, D. B. Campbell and M. C. Nolan, to F. Marchis and J. Berthier for their attempt to resolve Tama with, respectively, the Arecibo radiotelescope, and adaptative optics on a VLT. We also thank Philippe Bendjoya for helpful discussions and David Blair of the University of Western Autralia. Observations of Nyrölä observatory were made possible by a CCD grant by the AAVSO and the Curry Foundation. A part of this work was done within the Polish grant No. 1 P03D 00826.

\section{References}

Behrend, R. 2001, Orion, 304, 12

Behrend, R., Roy, R., Rinner, C., et al. 2004a, IAUC, 8265

Behrend, R., Roy, R., Sposetti, S., et al. 2004b, IAUC, 8292

Behrend, R., Roy, R., Sposetti, S., et al. 2004c, IAUC, 8354

Behrend, R., Bernasconi, L., Klotz, A., et al. 2004d, IAUC, 8389

Behrend, R., Roy, R., \& Sposetti, S. 2004e, Orion, 321, 20

Belton, M., \& Carlson, R. 1993, IAUC, 5948

Bendjoya, Ph., \& Zappalá, V. 2002, in Asteroids III, ed. W. F. Botke, A. Cellino, P. Paolicchi, \& R. P. Binzel (Univ. of Arizona), 613

Britt, D. P., Yeomans, D., Housen K, \& Consolmagno, G. 2002, Asteroid density, porosity and structure, in Asteroids III, Asteroids III, ed. W. F. Botke, A. Cellino, P. Paolicchi, \& R. P. Binzel, Univ. of Arizona, 485

Danby, J. 1992, in Fundamentals of celestial mechanisms (WillmannBell)

Harris, A. W., \& Lagerros, J. S. V. 2002, in Asteroids III, ed. W. F. Botke, A. Cellino, P. Paolicchi, \& R. P. Binzel (Univ. of Arizona), 207

Kaasalainen, M., \& Torppa J., 2001, Icarus, 153, 24

Kopal, Z. 1990, Mathematical theory of stellar eclipses (Dordrecht, Netherlands: Kluwer Academic Publishers)
Margot, J. L., Nolan, M. C., Negron, V., et al. 2003, IAUC, 8227

Merline, W. J., Weidenschilling, S. J., Durda, D. D., et al. 2002, in Asteroids III, ed. W. F. Botke, A. Cellino, P. Paolicchi, \& R. P. Binzel, Univ. of Arizona, 289

Michalowski, T., Bartczak, P., Velichko, F. P., et al. 2004, A\&A, 423, 1159

Minor Planet Center, http://cfa-www.harvard.edu/iau/mpc.htm

Monet, D. G. 1998, BAAS, 30, 1427

Sárneczky, K., Szabó, G., \& Kiss, L. L. 1999, A\&AS, 137, 363

1 Observatoire de Genève, 1290 Sauverny, Switzerland e-mail: Raoul.Behrend@obs.UniGe.CH

2 Association des Utilisateurs de Détecteurs Électroniques (AUDE), France

3 Observatoire des Engarouines, 84570 Mallemort-du-Comtat, France

${ }^{4}$ Observatoire de Blauvac, 84570 St-Estève, France

5 CESR, Observatoire Midi-Pyrénées, CNRS-UPS, BP 4346, 31028 Toulouse Cedex 04, France

${ }^{6}$ Observatoire de Haute Provence, 04870 Saint Michel l'Observatoire, France

7 IMCCE, 77 avenue Denfert-Rochereau, 75014 Paris, France

8 Observatoire de Bédoin, 47 rue Guillaume Puy, 84000 Avignon, France

9 Association T60, 14 avenue Édouard Belin, 31400 Toulouse, France

10 Astronomical Observatory, Copenhagen, Denmark

11 Observatoire de St-Jean de Bournay, 38440 St-Jean de Bournay, France

12 Observatoire Les Makes, G. Bizet 18, 97421 La Rivière, France

13 Agrupación Astronómica de Sabadell, PO Box 50, 08200 Sabadell, Spain

14 Shed of Science Observatory, 5213 Washburn Ave S., Minneapolis, MN-55410, USA

15 Planétarium de Saint-Étienne, 42000 Saint-Étienne, France

16 Sternwarte Weinheim, Klingenweg 14, 69469 Weinheim, Germany

17 Jakokoski Observatory, Univerity of Helsinki, PO Boy 14, 00014 Helsinki, Finland

18 Observatoire de Paris, 5 place Jules Janssen, 92195 Meudon, France

19 Max-Planck-Institut fuer Astronomie, Koenigstuhl 17, 69117 Heidelberg, Germany

20 Stazione Astronomica di Sozzago, 28060 Sozzago, Italy

21 Physics Dept., University of Rome Tor Vergata, Italy

22 Campo Catino Observatory, 03016 Guarcino, Italy

23 Ørsted DTU, Techn. Univ. of Denmark, Elektrovej, Building 327, 2800 Kgs. Lyngby, Denmark

${ }^{24}$ Nyrölä Observatory, Jyväskylän Sirius ry, Kyllikinkatu 1, 40100 Jyväskylä, Finland

${ }_{25}$ Carbuncle Hill Observatory, PO Box 946, Coventry, 02816, USA

${ }^{26}$ Darmstadt University of Technology, Magdalenenstrasse 4, 64289 Darmstadt, Germany

27 Observatorio di Gnosca, 6525 Gnosca, Switzerland

${ }^{28}$ DeKalb Observatory, 2507 CR 60, Auburn, 46706, USA

29 Observatoire François-Xavier Bagnoud, 3961 St-Luc, Switzerland

30 Observatori Astronómico de Mallorca, Camí de l'Observatori, s/n 07144 Costitx, Mallorca, Spain

31 CEAMIG-REA Observatory, 31545-12 Belo Horizonte, Brazil 